\title{
Imaginaire génétique : la critique face à la liste chez Jules Verne
}

Gaspard Turin

\section{(2) OpenEdition}

1 Journals

\section{Édition électronique}

URL : http://journals.openedition.org/genesis/3383

DOI : 10.4000/genesis.3383

ISSN : 2268-1590

\section{Éditeur :}

Presses universitaires de Paris Sorbonne (PUPS), Société internationale de génétique artistique littéraire et scientifique (SIGALES)

\section{Édition imprimée}

Date de publication : 15 décembre 2018

Pagination : 53-66

ISBN : 979-10-231-06282

ISSN : 1167-5101

\section{Référence électronique}

Gaspard Turin, « Imaginaire génétique : la critique face à la liste chez Jules Verne », Genesis [En ligne], 47 | 2018, mis en ligne le 15 décembre 2019, consulté le 21 janvier 2021. URL : http:// journals.openedition.org/genesis/3383 ; DOI : https://doi.org/10.4000/genesis.3383 


\section{Imaginaire génétique : la critique face à la liste chez Jules Verne}

Gaspard Turin

$\mathrm{I}$ 1 existe peu d'objets scripturaux aussi courants qu'une liste, se manifestant de manière aussi variée et dans un aussi grand nombre de contextes. Et il pourrait sembler étrange que la recherche académique, lorsqu'elle s'empare de cette forme (ce qu'elle a beaucoup fait ces dernières années ${ }^{1}$ ), ne cherche pas à la saisir de manière plus spécifique. Mais, alors que cela a été le cas par le passé2, il ne s'agit plus aujourd'hui de théoriser l'énumération, la série, ou encore la collection et le catalogue ${ }^{3}$ sans en référer en premier lieu au terme, central et commun, de liste. Cette forme apparaît comme suffisamment distincte du texte «ordinaire», soumis à la coordination de ses parties, pour être immédiatement identifiée, et les problématiques qu'elle soulève intéressent aujourd'hui la recherche bien au-delà des syntacticiens.

Une telle non-spécificité implique que le contexte de la préparation de l'œuvre littéraire n'est que l'un des contextes où la liste est susceptible de se manifester. Parmi ceux-ci, le rapport entre la phase préparatoire et le texte publié, lorsque celui-ci contient encore des listes, m'intéressera particulièrement. On pourrait dégager trois perspectives du constat de la présence de listes dans la genèse d'un texte littéraire : la première, sans doute la plus couramment envisagée, reviendrait à considérer cette forme comme source de production d'autre chose qu'elle-même - le texte «ordinaire», justement. Ce n'est pas la perspective qui sera suivie ici, mais les deux suivantes. La deuxième reviendrait à observer que la liste génétique produit encore de la liste dans le texte publié, mais que les deux sont différentes. La troisième consisterait à assimiler listes génétiques et listes publiées, à identifier les premières dans les secondes. Entre ces deux possibilités, l'enjeu se situe dès lors au niveau du processus d'élaboration de la liste : construite ou copiée.

On observera dans les pages qui suivent une situation critique particulière, où le rapport des textes publiés avec leur genèse putative pose un problème dont la liste est l'épicentre, justement à cause d'une confusion entre le copiage et la construction. En lisant les romans

1. En particulier Bernard Sève, De haut en bas. Philosophie des listes, Paris, Le Seuil, 2010; M. Lecolle, S. MilcentLawson et R. Michel (dir.), Liste et effet liste en littérature, Paris, Classiques Garnier, 2013; Umberto Eco, Vertige de la liste, Paris, Flammarion, 2009; Madeleine Jeay, Le Commerce des mots. L'usage des listes dans la littérature médiévale (XIIe-XVe siècles), Genève, Droz, 2006; Maya Lavault, «Aux listes, et cætera », Acta Fabula, vol. XIV, $\mathrm{n}^{\circ}$ 4, 2013, consultable en ligne; Gaspard Turin, Poétique et usages de la liste littéraire. Le Clézio, Modiano, Perec, Genève, Droz, 2017.

2. Par exemple, Madeleine Frédéric, «Énumération, énumération homologique, énumération chaotique. Essai de caractérisation », Stylistique, rhétorique et poétique dans les langues romanes, $\mathrm{n}^{\circ} 8$. Actes du XVIIe congrès international de linguistique et philologie romanes, Aix-en-Provence, Université de Provence, 1986, ou Béatrice Damamme-Gilbert, La Série énumérative. Étude linguistique et stylistique s'appuyant sur dix romans français publiés entre 1945 et 1975, Genève, Droz, 1989.

3. Voir à ce sujet Bernard Vouilloux, «Le discours sur la collection», Romantisme, n 112, 2001, p. 103 : «La cellule nucléaire du catalogue est $[\ldots]$ la liste». 
de Jules Verne, une grande partie de la critique semble en effet considérer que les listes dont ils sont parsemés trouvent leur origine dans une mauvaise gestion des sources documentaires de l'écrivain : ces listes sont jugées sévèrement, soit parce qu'elles auraient été simplement recopiées, soit parce qu'elles témoigneraient uniquement d'une nécessité d'intégrer au roman des informations de nature didactique, soit parce qu'elles seraient tout simplement ennuyeuses à lire - le plus souvent tout cela à la fois.

On verra que ce jugement est discutable. Ce qu'on interrogera ici, plus sur la base d'un corpus critique que de littérature primaire, est donc un geste critique. La liste est un objet fréquent dans la préparation d'un texte littéraire, comme elle l'est d'ailleurs dans la préparation d'autres activités (achats, recettes de cuisine, expéditions en pays inconnu, etc.). C'est probablement la raison pour laquelle, dans un cas comme celui de Verne, il est difficile d'imaginer sa présence dans la préparation du roman sous la forme d'un recopiage : elle devrait être, comme ailleurs, un instrument de construction. Elle apparaît dès lors particulièrement propice à l'éveil d'un imaginaire génétique : une rétroprojection sur la genèse de l'œuvre à partir du texte, servie i) par un état particulier du texte publié, suggérant une certaine lisibilité des étapes de sa création, et ii) par un dossier génétique incomplet, permettant à l'hypothèse de remplacer le phénomène.

Un espace d'incertitude Considéré sous l'angle de ses textes publiés, Verne peut très certainement correspondre à génétique la qualification d'écrivain «à listes »; en témoigne notamment le travail de Philippe Hamon sur les descriptions de Vingt mille lieues sous les mers, nourries «d'un vocabulaire spécialisé à la fois manifesté sous forme de listes, listes de noms de peintres, de noms de coquillages, de noms de poissons $[\ldots]^{4}$ » - c'est-à-dire de listes à valeur documentaire. En ce qui concerne l'entrée génétique dans l'œuvre, je m'appuierai sur les travaux d'autres spécialistes, en particulier Philippe Scheinhardt et William Butcher, sachant qu'à ma connaissance il n'existe pas d'étude spécifique sur la question de la liste chez Verne sous l'angle génétique. Et pour cause : les états préliminaires à la rédaction des manuscrits, qui auraient permis de dévoiler l'archéologie des listes publiées, n'existent plus, dans leur très grande majorité. «N'ont été conservées en général que les mises au net corrigées avant épreuves ${ }^{5}$.» Il n'existe pas de rapport complet concernant le contenu matériel précis de ces états préliminaires, bien qu'on dispose d'informations à propos de certains stades de l'écriture romanesque. Butcher présente comme l'un des stades préliminaires de l'écriture de Verne celui d'un rassemblement d'«observations, détaillées et plutôt fastidieuses, glanées lors d'une lecture en vue d'un roman précis, concernant un pays particulier, [...] extraites des études géographiques. [...] Passent par ce système sans doute la quasi-totalité des informations extérieures. ${ }^{6}{ }$ On sait aussi que Verne disposait d'un jeu de «fiches» monographiques, dont Alain Buisine observe qu' elles semblent réapparaître telles quelles dans les romans, tout en s'assimilant à un listage (il tient la rupture stylistique

\footnotetext{
4. Philippe Hamon, Du Descriptif, Paris, Hachette Supérieur, 1993, p. 220. Voir aussi, du même auteur, La Description littéraire, Paris, Macula, 1991, p. 200.

5. Philippe Scheinhardt, «Jules Verne, un processus d'écriture sous contraintes », Genesis, n 33, 2011, p. 173-186 :

p. $174, \S 4$, consultable en ligne.

6. William Butcher, Jules Verne inédit : les manuscrits déchiffrés, Lyon, ENS Éditions, 2015, p. 59.
} 
comme indice d'une tendance à la liste) ${ }^{7}$. Enfin, Verne lui-même avoue que son rapport à la documentation est intimement lié aux formes encyclopédiques : «J'ai eu la chance d'entrer dans le monde à un moment où il existait des dictionnaires sur tous les sujets possibles. Il me suffisait de trouver dans le dictionnaire le sujet sur lequel je cherchais un renseignement, et voilà8.» Un «et voilà» pouvant suggérer par litote qu'un forfait a eu lieu, et que ce forfait est de l'ordre du recopiage... Dans la mesure surtout où de tels documents s'apparentent déjà, dans leur organisation formelle, à la liste. Que l'on ajoute à ces éléments la présence intensive de la liste dans les moments de préparation de très nombreux romans, et les vecteurs se multiplient qui laissent croire à une similitude complète entre listes préparatoires et publiées.

Précisons encore deux hypothèses concernant la phase prérédactionnelle chez Verne. D'une part il semblerait que la liste n'ait pas joué de rôle de planification pour l'ensemble de l'œuvre, selon Scheinhardt : «Rappelons la liste des romans faits et à faire établie pour mémoire par Honoré de Balzac pour la programmation des chantiers génétiques de la Comédie humaine. Rien de tel dans la fabrique des Voyages extraordinaires ${ }^{9}$. D D'autre part quelques indices laissent à penser qu'elles ont pu jouer ce rôle dans la planification locale des œuvres : Butcher signale qu'il subsiste «trois documents indicatifs, préparatoires au roman inachevé Jédédias Jamet (1847), dont deux sous forme de listes : une généalogie du héros éponyme et un itinéraire de Tours à Rotterdam 10 ». Puis, du manuscrit du Tour du monde en 80 jours, en particulier de ses marges où figurent des listes, il déduit qu'elles constituent l'essentiel de la construction du roman vernien : il s'agirait là de «l'état le plus ancien de la création, dans lequel Verne construit son roman, sous forme de listes $11 »$. Mais Butcher n'établit pas de lien entre ces listes et celles, de nature descriptive, que l'on trouve dans l'ouvrage publié. Il n'est, en somme, pas possible de déduire de ces informations la présence ou l'absence de listes à valeur documentaire durant cette phase. Un espace d'incertitude se maintient donc, laissant le champ libre à une hypothèse dont la plausibilité reste inentamée : parmi les listes documentaires établies par Verne, résultat du «glanage» dont parle Butcher, certaines auraient subsisté jusque dans les versions publiées des romans.

Le soupçon d'une écriture constituée par un montage trop visible de listes documentaires apparaît à la lecture de certains épisodes des Voyages extraordinaires, dans lesquels la liste transgresse l'économie narrative du roman d'aventures. Des travaux ayant présidé à l'écriture de Cinq semaines en ballon, le premier roman du cycle, presque rien n'a été conservé. Mais il est tentant de voir dans cet extrait, tiré des premières pages où l'expédition de Samuel Fergusson se prépare, un recopiage pur et simple :

Des toasts nombreux furent portés [...] aux célèbres voyageurs qui s'étaient illustrés sur la terre d'Afrique. On but à leur santé ou à leur mémoire, et par ordre alphabétique, ce qui est très anglais :

7. «La fiche s'insère dans le développement romanesque [...]. C'est ainsi que s'effectu[e] [...] un passage de phrases très riches en expansions (tout particulièrement des relatives) à une écriture sèche, simple juxtaposition d'indépendantes.» Alain Buisine, «Un cas limite de la description : l'énumération», dans Ph. Bonnefis et P. Reboul (dir.), La Description. Nodier, Sue, Flaubert, Hugo, Verne, Zola, Alexis, Fénéon, Lille, Presses universitaires de Lille, 1981, p. 86

8. Jules Verne en entretien, cité par Philippe Scheinhardt, art. cit., p. 174, § 6.

9. Philippe Scheinhardt, art. cit., p. $172, \S 2$.

10. William Butcher, op. cit., p. 61.

11. Ibid., p. 294. 
à Abbadie, Adams, Adamson, Anderson, Arnaud, Baikie, Baldwin, Barth, Batouda, Beke, Beltrame, du Berba, Bimbachi, Bolognesi, Bolwik, Bolzoni, Bonnemain, Brisson, Browne, Bruce, BrunRollet, Burchell, Burckhardt, Burton, Caillaud, Caillié, Campbell, Chapman, Clapperton, Clot-Bey, Colomieu, Courval, Cumming, Cuny, Debono, Decken, Denham, Desavanchers, Dicksen, Dickson, Dochard, Duchaillu, Duncan, Durand, Duroulé, Duveyrier, Erhardt, d'Escayrac de Lauture, Ferret, Fresnel, Galinier, Galton, Geoffroy, Golberry, Hahn, Halm, Harnier, Hecquart, Heuglin, Hornemann, Houghton, Imbert, Kaufmann, Knoblecher, Krapf, Kummer, Lafargue, Laing, Lajaille, Lambert, Lamiral, Lamprière, John Lander, Richard Lander, Lefebvre, Lejean, Levaillant, Livingstone, Maccarthie, Maggiar, Maizan, Malzac, Moffat, Mollien, Monteiro, Morrisson, Mungo-Park, Neimans, Overwev, Panet, Partarrieau, Pascal, Pearse, Peddie, Peney, Petherick, Poncet, Prax, Raffenel, Rath, Rebmann, Richardson, Riley, Ritchie, Rochet d'Héricourt, Rongawi, Roscher, Ruppel, Saugnier, Speke, Steidner, Thibaud, Thompson, Thornton, Toole, Tousny, Trotter, Tuckey, Tyrwitt, Vaudey, Veyssière, Vincent, Vinco, Vogel, Wahlberg, Warington, Washington, Werne, Wild, et enfin au docteur Samuel Fergusson qui, par son incroyable tentative, devait relier les travaux de ces voyageurs et compléter la série des découvertes africaines ${ }^{12}$.

De fait, il paraît probable à bien des critiques que les listes présentes dans les textes ne soient rien d'autre que la reproduction du matériau réuni lors de la phase de documentation, à l'instar de Simone Vierne :

Jules Verne recopie [...], avec conscience mais sans grande modification, les renseignements qu'il puise dans les publications scientifiques de l'époque. Cela prend l'allure d'énumérations, fort longues, sans aucune vergogne, et il faut bien avouer que tout lecteur moyen «saute» ces passages 13 .

Jacques Noiray, quant à lui, juge également sévèrement la présence de listes dans le roman vernien :

Les longues énumérations de noms savants que nous avons étudiées comme des procédés d'acclimatation du savoir naturaliste dans le texte littéraire apparaissent aussi comme des moyens désinvoltes de se débarrasser d'une documentation trop pesante, en exhibant jusqu'à l'absurde un mandat didactique qui n'a jamais été accepté sans réticence ${ }^{14}$.

Voici une explication partagée par Alain Buisine, qui voit l'énumération vernienne comme une fonction de sa pratique descriptive, laquelle «est très exactement celle que signifie le latin describere, source étymologique du mot description, initialement utilisé au sens de transcribere. Describere et transcribere, c'est transcrire, copier, selon une pratique qui met en rapport deux textes ${ }^{15}$.» Pour Buisine toujours, comme on l'a vu, la présence de la liste dans le roman vernien est le résultat de l'insertion de «fiches » de lecture; celles-ci traversent les phases de préparation jusqu'au roman publié, le procédé étant apparenté à une «pilule didactique $^{16}$ », plus facile à avaler si elle se présente sous la forme compacte d'une liste. Citons encore Marie-Hélène Huet, qui dans la notice qu'elle consacre au Testament d'un

12. Cinq Semaines en ballon, Hachette, 1978 [1863], p. 13.

13. Simone Vierne, Jules Verne et le Roman initiatique, Lille, Service des reproductions des thèses, 1972, p. 574-575. 14. Jacques Noiray, «L'inscription de la science dans le texte littéraire : l'exemple de Vingt mille lieues sous les mers », dans C. Reffait et A. Schaffner (dir.), Jules Verne ou les inventions romanesques, Amiens, Encrage Universités, 2007 , p. $48-49$.

15. Alain Buisine, art. cit., p. 82.

16. Ibid., p. 88. 
excentrique emprunte à Michel Serres la formule selon laquelle les descriptions meublant le roman seraient «d'un ennui épais», et poursuit :

De même qu'autrefois les longues énumérations dont Conseil gratifiait les lecteurs de Vingt mille lieues sous les mers, ces chiffres austères, entièrement étrangers au déroulement de l'action, scandent les itinéraires des joueurs comme autant de bornes kilométriques dans la vitesse des déplacements ${ }^{17}$.

Enfin, Jean-Paul Sartre lui-même observait, dans Les Mots, le décalage problématique à l'œuvre chez Verne, entre le mandat didactique et la progression romanesque :

Boussenard et Jules Verne ne perdent pas une occasion d'instruire : aux instants les plus critiques, ils coupent le fil du récit pour se lancer dans la description d'une plante vénéneuse, d'un habitat indigène. Lecteur, je sautais ces passages didactiques; auteur, j'en bourrai mes romans; je prétendis enseigner à mes contemporains tout ce que j'ignorais : les mœurs des Fuégiens, la flore africaine, le climat du désert. Séparés par un coup du sort puis embarqués sans le savoir sur le même navire et victimes du même naufrage, le collectionneur de papillons et sa fille s'accrochaient à la même bouée [...]. Les malheureux échapperaient-ils à la mort? J'allais chercher le tome «Pr-Z» du Grand Larousse, je le portais péniblement jusqu'à mon pupitre, l'ouvrais à la bonne page et copiais mot pour mot en passant à la ligne : «Les requins sont communs dans l'Atlantique tropical. Ces grands poissons de mer très voraces atteignent jusqu'à treize mètres de long et pèsent jusqu'à huit tonnes... » Je prenais tout mon temps pour transcrire l'article : je me sentais délicieusement ennuyeux $[\ldots]^{18}$.

Les procédés stylistiques employés par Verne apparaissent comme extérieurs à la rédaction romanesque proprement dite, ce qui induit qu'ils sont également antérieurs à elle. La critique observe facilement dans la liste vernienne un indice exogénétique, parce que la liste lui apparaît comme un procédé facile, non stylisé, voire non écrit, tout entier du côté de la «didactique » et de ses genres, et ne tenant pas compte de l'économie narrative du roman. Ceci occasionne un jugement de valeur négatif, portant sur la «longueur» ou l'«ennui»: en somme, un manque d'intérêt pour une production littéraire n'obéissant pas à une élaboration scripturale pensée de préférence comme endogénétique. En témoigne le ton globalement narquois quant à la qualité de l'écriture, lorsque celle-ci est associée à une liste qui, présente dans les versions publiées des romans, ne s'explique que comme une mauvaise intégration de la documentation, une survivance de celle-ci.

La séparation à l'œuvre entre «littérature » et «didactique» rejoint le positionnement éthique de Verne face à son travail, tel qu'il a été observé par Scheinhardt. À l'en croire, ce positionnement est double : l'un est public et l'autre privé. L'ethos public (proche de ce que Jérôme Meizoz appellerait «la posture») est celui qui transparaît des entretiens donnés par Verne de son vivant. Il s'y présente comme un écrivain professionnel, muni donc des attributs habituels de la profession : espaces de travail particuliers, rituels d'écriture, protocoles immuables entre la conceptualisation et la rédaction des romans. Même si «Jules Verne ne

\section{Une intégration mal négociée}

17. Marie-Hélène Huet, «Notice» dans Jules Verne, Voyage au centre de la terre et autres romans, Paris, Gallimard, «La Pléiade», 2016, p. 1296

18. Jean-Paul Sartre, cité par Timothy A. Unwin, Jules Verne : Journeys in Writing, Liverpool, Liverpool University Press, 2005, p. 180 
peut envisager la création littéraire qu'à l'intersection de l'art et du métier», il «s'efforce [...] de se positionner comme artiste ${ }^{19}$ », c'est-à-dire comme responsable de sa propre production, selon un idéal de création autonome hérité du romantisme.

Un idéal toutefois remis en cause par le second aspect de l'ethos vernien, lié à sa situation éditoriale. Celle-ci, restreinte par Verne à la sphère privée (une manière de dire qu'il préférait que cela ne se sache pas), en réduit fortement le principe d'un modus operandi individuel, puisque la collaboration Verne-Hetzel s'avère le lieu d'une influence, voire d'une ingérence de l'éditeur, dont la «tutelle dériv[e] vers le contrôle du pouvoir créatif 20 ». Rappelons ici le caractère particulier du contrat qui liait Verne à son éditeur : il s'agit tout d'abord pour Verne, dès 1862 (c'est-à-dire au début des Voyages extraordinaires), de fournir à Hetzel «chaque année trois volumes composés dans le genre de ceux qu'il a précédemment édités » (chiffre réduit à deux volumes en 1871) ${ }^{21}$. Puis l'éditeur formule également, quelques années plus tard, des précisions concernant ce «genre» : il s'agit pour Verne «de résumer toutes les connaissances géographiques, géologiques, physiques, astronomiques, amassées par la science moderne, et de refaire, sous la forme attrayante et pittoresque qui lui est propre, l'histoire de l'univers $22 »$. De telles exigences impliquent un rythme de travail conséquent pour le romancier et un contrôle considérable de la part de l'éditeur.

Dans sa très vaste étude des manuscrits verniens, Butcher se préoccupe beaucoup de rendre compte des responsabilités scripturales en jeu. Son but étant de rétablir le roman vernien dans une pureté qu'il estime fondamentale, il attaque très violemment Hetzel, considérant ses interventions en cours d'écriture parfaitement illégitimes. Butcher les qualifie d' 'éléments allogènes 23 », impose un regard manichéen sur la situation («[1]es émotions usées et insincères [de Hetzel] ne reflètent certainement pas l'esprit authentique de Verne $24 »)$ et propose même de voir dans chaque maladresse d'écriture la trace de l'éditeur :

[C]haque passage étranger au génie de Verne doit receler bien d'autres éléments inauthentiques [...].

Le doute s'installe ainsi concernant chaque animal, chaque technologie, chaque happy end, chaque sentiment un peu forcé, chaque plaidoyer pro domo, chaque insincérité, chaque phrase quelque peu boiteuse, chaque épisode légèrement bancal, chaque trame inconsciemment estropiée 25.

Le vrai, l'unique Verne, «à l'état pur ${ }^{26}$ », celui de la «nature 27 », n'apparaîtrait qu'à condition qu'on le débarrasse enfin de l'éditeur scélérat. L'intérêt pour nous, dans cette lecture d'un processus d'écriture qui ne serait authentique qu'à la condition d'être individuel, réside dans le fantasme romantique qu'elle laisse transparaitre : celui d'un auteur rendu à un génie créateur, à une pureté démiurgique.

19. Philippe Scheinhardt, art. cit., respectivement p. 175 et p. $176, \S 7$ et $\S 12$.

20. Ibid., p. 178, § 17.

21. Cité par Daniel Compère, Jules Verne écrivain, Genève, Droz, 1991, p. 17.

22. Ibid., p. 20.

23. William Butcher, op. cit., p. 435.

24. Ibid., p. 448.

25. Ibid., p. 442

26. Ibid., p. 442.

27. Ibid., p. 14. 
Dans l'anathème qui l'occupe, Butcher ne parle pratiquement pas de la composition vernienne de la liste, hormis dans un commentaire lapidaire à propos de la bibliothèque de Nemo, dont les états successifs indiquent selon lui qu'à la liste (des ouvrages et des auteurs) tend à se substituer une séquence rédigée de manière plus traditionnelle : «Tout se passe comme si Verne était tout à ses artistes, comme si son cœur n'y était pas pour faire une grande énumération scientifique, dont la présence finale serait due à l'influence, ou à la pression, de Hetzel28. ${ }^{28}$ La liste vernienne, comme le suggère cette remarque, pourrait être, elle aussi, mise sur le compte de l'ingérence éditoriale. Elle serait dès lors disqualifiée au profit de son développement, traitement littéraire plus conforme, selon Butcher, aux affinités électives que Verne doit nécessairement développer avec «ses artistes». Mais quoi qu'il en soit, le regret d'ensemble que Butcher formule quant aux défauts des Voyages extraordinaires rejoint l'avis des critiques, selon lequel les listes des romans de Verne seraient le résultat d'une intégration mal négociée, car fondée sur des nécessités «didactiques». Entre la déploration d'un Verne estropié par son éditeur et le dédain face à une intégration maladroite des exigences pédagogiques contractuelles, on retrouve deux exemples d'une disposition de la critique à voir dans la liste une défaite de l'art face aux impératifs triviaux de l'artisanat, une transmission du savoir plutôt qu'une expression poétique.

Avant de revenir à cette disposition, il s'agit de nuancer la vision critique exposée ci-dessus : contrairement aux suppositions plus ou moins explicites qui y sont formulées, prétendre que la liste présente dans les romans de Verne serait la trace d'un recopiage littéral est aller trop vite en besogne, et témoigne plutôt d'une croyance que d'une réalité. Il pourrait en être autrement.

On sait que les sources de Verne, outre les dictionnaires et les encyclopédies, font la part belle aux ouvrages de vulgarisation scientifique ${ }^{29}$. Et lorsque, faute d'accès aux documents préparatoires, la critique se rend attentive à la nature précise des emprunts de Verne à ses sources, elle identifie sans équivoque l'importance prépondérante de tels ouvrages vulgarisateurs. Je garderai l'exemple du roman de Verne le plus souvent cité pour la quantité de listes qu'il contient, Vingt mille lieues sous les mers. L'appareil critique de l'édition «Pléiade 30 » ne semble pas pris en défaut lorsqu'il s'agit d'identifier des emprunts et des recopiages mais ne signale jamais, en ce qui concerne les listes, de copie de dictionnaires ou d'encyclopédies. Au contraire, les passages où se reconnaissent des échos directs à la documentation prérédactionnelle se rapportent aux ouvrages de Frédol, Michelet ou Mangin dont la textualité n'est pas de nature listique, mais bien plutôt narrative. Sur les 256 notes relatives aux deux volumes de Vingt mille lieues sous les mers, 51 renvoient à des sources documentaires de vulgarisation ou à des articles de dictionnaires, tandis que seulement 6 s'attachent à commenter des listes. L'une d'entre elles porte sur le mot du domestique Conseil, au départ du voyage, celui-ci s'étonnant que «les archiotherium, les hyracotherium, les oréodons, les chéropotamus

28. Ibid., p. 184.

29. Notamment La Mer de Jules Michelet, Les Mystères de l'océan d'Arthur Mangin et Le Monde de la mer d'Alfred Frédol : voir à ce propos Jacques Noiray, art. cit., p. 30 sq.

30. Jules Verne, Voyages extraordinaires. Les Enfants du capitaine Grant, Vingt mille lieues sous les mers, Paris, Gallimard, «La Pléiade», J.-L. Steinmetz (dir.), 2012. La référence concerne ici les «notes » consacrées au second roman : p. 1355-1388. 
et autres carcasses de Monsieur ${ }^{31} »$ ne fassent pas partie de l'expédition; la note explique de quels animaux il s'agit et, pour le dernier d'entre eux uniquement, donne deux sources documentaires possibles.

Il existerait un contre-exemple, une liste de Voyage au centre de la terre, dont la source reconnue 32 pourrait indiquer qu'elle fut effectivement «recopiée»: «Qu'ils [les terrains rencontrés par les explorateurs] fussent pliocènes, miocènes, éocènes, crétacés, jurassiques, triasiques, permiens, carbonifères, dévoniens, siluriens ou primitifs, cela me préoccupa peu ${ }^{33}$.» L'ouvrage-source, dont l'extrait est reproduit en figure 1, est à nouveau un ouvrage de vulgarisation. Mais on remarquera qu'il provient d'un objet hybride, illustration et tableau tout à la fois. S'agit-il d'un maintien tel quel de la liste ou d'une transformation de celle-ci (une linéarisation de l'illustration-tableau à la liste) ? La nature de cet emprunt est discutable, surtout dans la mesure où la succession des ères géologiques est un fait scientifiquement incontestable à l'époque de Verne, qui aurait pu trouver cette information ailleurs. Cette remarque implique dès lors une précision argumentative : il s'agit moins, ici, de vérifier la source effective des informations scientifiques que Verne utilise, que d'observer les lectures du texte vernien et l'évaluation qu'elles entrainent. Que les méthodes utilisées pour envisager de telles lectures ne soient pas celles de la critique génétique n'empêche pas qu'il y ait là un enjeu de nature génétique.

Il y a bien, de la part de la critique, une équivalence trop rapidement établie entre les listes publiées, perçues comme ennuyeuses, et le recopiage appliqué et laborieux qui devrait, pour elle, en constituer le geste-source. Mais le problème réside plutôt dans l'usage inconsidéré d'une certaine idée de la genèse du roman vernien par le discours évaluatif de la critique. D'un point de vue génétique, l'essentiel de la rédaction vernienne de listes pourrait bien plutôt correspondre à une transformation des sources convoquées pour les établir. Une transformation qui ne se confinerait pas à un seul niveau de préparation : ainsi on peut imaginer que Verne ait recopié des listes qu'il aurait lui-même composées, mais il est également très probable qu'il n'ait jamais cessé de les recomposer et de les transformer au fil de son travail. D'une écriture de liste dont on pourrait attendre qu'elle reste confinée aux premiers stades de l'écriture, Verne fait usage jusqu'à la maintenir dans les romans publiés. Les raisons en seraient multiples ; d'une part, comme le souligne Timothy Unwin, il s'agit pour l'auteur de satiriser sa propre pratique d'écriture scientifique :

It is certainly the case [...] that the discourses of science and positivism invade the Vernian text and appear as foreign bodies within the fiction. Lists, taxonomies and inventories [...] may on occasions seem spectacularly recalcitrant to narrativisation. Yet part of the point of Verne's lists is to take the language of scientific precision to extremes which display it within the fiction, showing up its folly and its absurdity 34 .

On se souvient que Noiray allait dans le même sens lorsqu'il parlait d'un «mandat didactique exhibé jusqu'à l'absurde» - encore manquait-il de faire d'une telle exhibition le lieu

31. Ibid., p. 748.

32. Jean-Luc Steinmetz, «Notes», dans Jules Verne, Voyage au centre de la terre et autres romans, Paris, Gallimard,

«La Pléiade», J.-L. Steinmetz (dir.), 2016, p. 1157.

33. Jules Verne, Voyage au centre de la terre, op. cit., p. 105.

34. Timothy A. Unwin, op. cit, p. 204. 


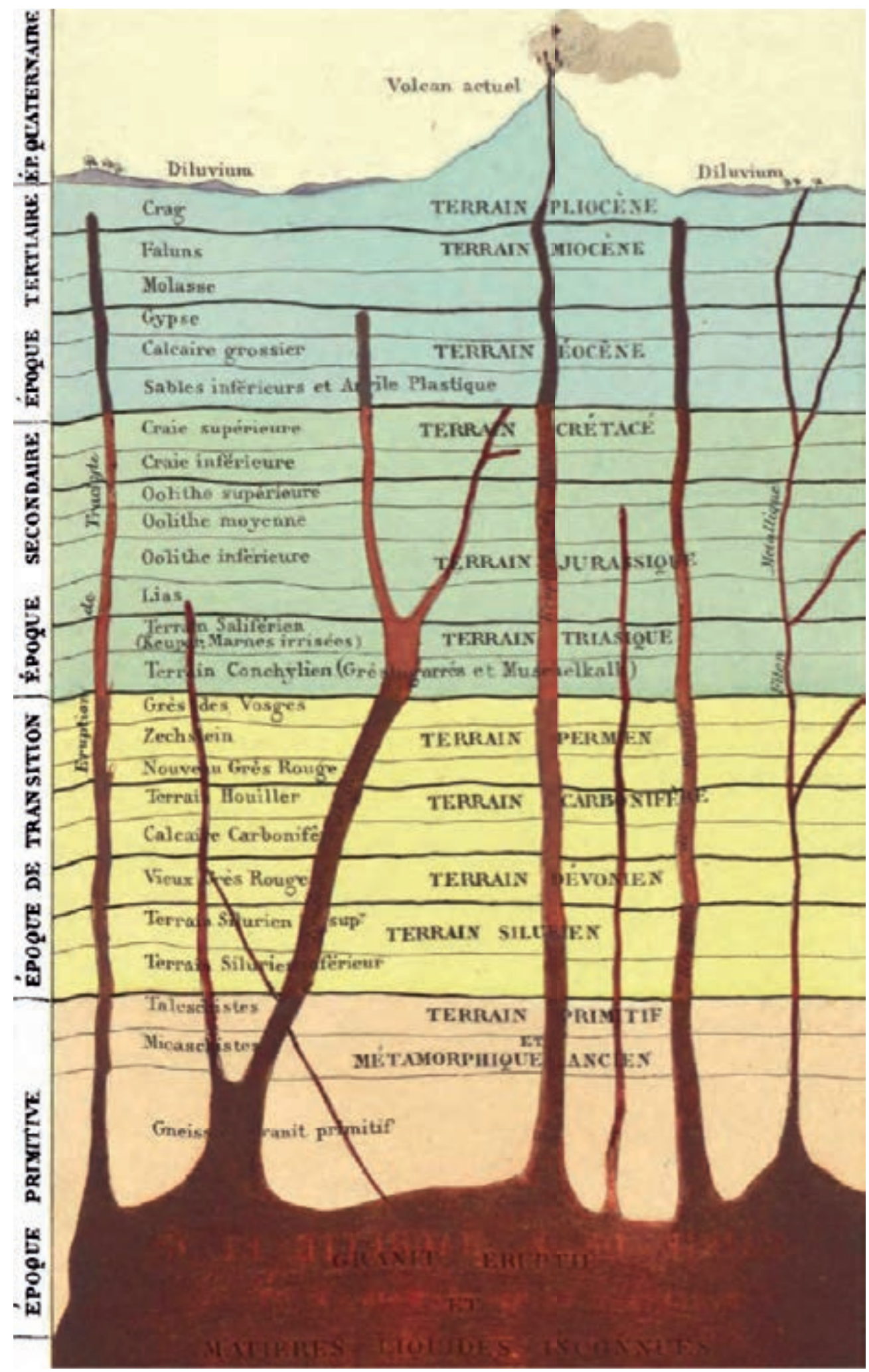

Fig. 1 : «Coupe idéale de l'écorce solide du globe terrestre», Louis Figuier, La Terre avant le Déluge, Paris, Hachette, 1863. Source : IRIS, bibliothèque numérique en histoire des sciences, consultable en ligne. 
d'une qualité poétique assumée, plutôt que d'une faiblesse stylistique, d'une « désinvolture ». La liste-satire est d'ailleurs nettement pourvue de signes d'ironie, ce dont témoigne le discours d'accompagnement de la liste citée plus haut : «on but à leur santé ou à leur mémoire, et par ordre alphabétique, ce qui est très anglais». Le systématisme proche de l'absurde, consistant à lever son verre à chaque nom cité - geste qui convoque le double cliché sociologique de la raideur et de l'alcoolisme de l'Anglais - nourrit une ironie manifeste, qui porte également sur le listage scientifique, ici fourni selon un ordre (l'alphabet) parfaitement détaché de l'éventuel bénéfice qu'un tel épisode pourrait fournir dans le contexte d'une préparation à une expédition. Il fallait, pour un tel bénéfice, que l'ordre de présentation fût historique; faute de quoi la liste apparaît «hors sol», détachée aussi bien de l'économie narrative que pédagogique mise en place dans l'ensemble des Voyages extraordinaires. Elle se trouve dès lors mise en évidence, outrée, et non maladroite. Verne ne fait pas que reprendre les informations documentaires: il mime également, au passage, les codes d'archivage encyclopédiques.

D' autre part, le maintien de la liste correspond à la rémanence d'un désir (lié à ses fonctions : classement, récapitulation, renouvellement de la vision) qu'il convient de transmettre. Perec, un siècle plus tard, identifiera explicitement ce désir : "L'écriture contemporaine, à de rares exceptions (Butor) a oublié l'art d'énumérer : les listes de Rabelais, l'énumération linnéenne des poissons dans Vingt mille lieues sous les mers, l'énumération des géographes ayant exploré l'Australie dans Les Enfants du capitaine Grant... ${ }^{35} »$ Un tel témoignage présente aussi une dimension axiologique, opposée à l'ordinaire fraîcheur d'accueil de la liste vernienne par la critique : il s'agit, pour Perec, de défendre un plaisir de lecture, une valeur littéraire positive de la liste.

Deux modalités interprétatives de la liste vernienne s'opposent donc ici. Soit la liste passe pour un défaut de méthode, une forme propre à rendre dans le roman l'impératif didactique contractuel, mais impropre à maintenir le récit dans son économie narrative et son efficacité fictionnelle. Soit elle présente un bénéfice, double : celui d'une distance critique envisagée comme une réaction volontaire à cette même efficacité; celui d'une valorisation du plaisir classificatoire. L'avantage de la seconde modalité de lecture sur la première réside dans le fait qu'elle réconcilie exo- et endogenèse dans le regard porté sur le rôle de la liste dans la préparation du roman.

Imaginaire génétique : cosmogonie et palingénésie
Il n'aura, je l'espère, pas échappé à mon lecteur que, par les lignes qui précèdent, j'ai essentiellement substitué une hypothèse de lecture à une autre. L'exercice aura surtout eu comme but d'exemplifier comment, face à l'absence d'un dossier génétique complet, le vide qui se présente à l'occasion du déficit documentaire sera facilement comblé par le recours à un imaginaire génétique - peut-être ce recours est-il même inévitable. L'explication de la liste génétique vernienne, à défaut de documents sur lesquels s'appuyer, aura longtemps pris le pli d'un imaginaire particulier, tributaire d'une conception romantique plus ou moins prononcée de l'art et de la construction de l'œuvre littéraire comme imputable à un génie individuel. Au passage on remarquera qu'une telle lecture était déjà programmée par Verne, non seulement dans son ethos public, comme nous l'avons déjà vu, mais aussi dans le choix de ne conserver

35. Georges Perec, Penser/Classer, Paris, Le Seuil «La Librairie du XXe siècle», 2005 [1985], p. 21. 
que ses manuscrits définitifs. Comme l'écrit Pierre-Marc de Biasi, «dans sa quasi-perfection finale, que confirment quelques infimes modifications de dernière minute, le manuscrit définitif paraît donner raison au mythe de l'écriture inspirée qui serait un don du Ciel ${ }^{36}$ ».

S'il est inconfortable, dans un épistémè romantique prolongé par la critique contemporaine, de penser qu'un texte de fiction se trouve parasité par des séquences tirées d'autres textes, cet inconfort est redoublé dans le cas où ces séquences sont des listes, par l'habitude consistant à considérer cette forme comme relevant de la genèse de la création littéraire. Une habitude perceptible dans cette citation de Hamon :

[La liste] semble être par exemple, quand on la rencontre dans les brouillons préparatoires de l'écrivain, où elle foisonne, du côté de l'avant- ou de la pré-littérature : forme et moment archaïque de la création, signe d'un projet, signe d'un programme, sorte de prospectus demandant à entrer en fiction 37 .

Ainsi que dans cette réflexion de Jacques Neefs :

La liste, le répertoire, le quadrillage (religieux, administratifs, juridiques) semblent être constitutifs de l'écriture. Faire œuvre dans l'espace de cette origine de l'écriture est peut-être l'impératif plus ou moins dissimulé, plus ou moins conscient, plus ou moins joué, qui détermine l'écriture créatrice et la dimension esthétique des œuvres de langage 38 .

De telles déclarations ne sont évidemment pas erronées, mais elles nourrissent un mythe, celui d'un début qui ne serait précédé par rien. Mythe largement partagé dans le cas de la liste, fréquemment perçue comme une sorte de «degré zéro 39 »- mais un degré zéro qui confond, dans le cas de la critique vernienne, le négatif du texte inabouti (voire d'un «non-texte en attente de devenir texte $40 »$ ) et le positif d'une écriture en perpétuel devenir. Ce mythe est nourri par un sentiment diffus, presque universel, dont les récits bibliques seraient un substrat : en particulier la Genèse et sa double liste inaugurale (1:1 et 2:20), où Dieu fait advenir toutes choses par leur nomination énumérée, après quoi les animaux qui défilent devant Adam reçoivent de lui leurs noms. De manière plus inattendue, l'anthropologie n'est pas non plus totalement étrangère à cette fascination; Jack Goody, dans sa magistrale étude de l'apparition de l'écriture comme technologie intellectuelle, fait coïncider les premières apparitions historiques de l'écriture avec la liste : «Faire des tableaux ou des listes [...] est très caractéristique des premiers systèmes d'écriture ${ }^{41}$.» Pour en revenir à l'écriture comme acte individuel de création, on sait bien que «l'origine de l'écriture est toujours ailleurs,

36. Pierre-Marc de Biasi, Génétique des textes, Paris, CNRS Éditions «Biblis», 2011, p. 31.

37. Philippe Hamon, Puisque réalisme il y a, Genève, La Baconnière, 2015, p. 163.

38. Jacques Neefs, préface, dans B. Didier et J. Neefs (dir.), Penser, classer, écrire : de Pascal à Perec, Saint-Denis, Presses universitaires de Vincennes, 1990, p. 10 (je souligne).

39. L'expression est empruntée à Jean-Michel Adam, pour qui l'énumération constitue «le degré zéro de la description». Les Textes : types et prototypes, Paris, Nathan, 1992, p. 81.

40. Philippe Hamon, Puisque réalisme il y a, op. cit, p. 163.

41. Jack Goody, La Raison graphique. La domestication de la pensée sauvage, Paris, Minuit, 1979 [1977], p. 141. Précisons que Goody ajoute aussitôt ne pas «penser pour autant que le fait de fabriquer des tableaux, des listes et des formules ait pour origine unique l'avènement de l'écriture», observant une corrélation sans forcer la causalité des documents archéologiques sur 1'histoire, sans prétendre identifier formellement la liste comme «la» première forme d'écriture de l'humanité. 
inatteignable, antérieure à tout geste scriptural 42 »... Mais ce savoir n'empêche pas la séduction édénique d'une croyance en un point de concordance, un lieu géométrique incarné ici par la liste, origine de tout. Un point vers où se dirigerait le regard rétrospectif de l'imaginaire génétique, vers où convergeraient les lignes d'une perspective commune à l'histoire des formes et à celle de la pensée humaine.

Face à cette tentation cosmogonique de la liste, l'étude de Verne permet de substituer une autre explication, non moins mythique, puisqu'elle ne fait que remplacer un imaginaire génétique par un autre, mais proposant néanmoins une alternative féconde. On pourrait la qualifier de palingénésique : au concept d'une création ex nihilo s'oppose celui d'une création perçue comme un éternel retour, un cycle. Dans ce second paradigme, la liste génétique devient le signal d'autre chose : elle ne désigne pas les items énumérés comme une apparition (une invention), mais comme une réapparition (une répétition), qui reconditionne l'ensemble des Voyages extraordinaires. Elle suppose un avant-discours, que l'on réitère à raison et non comme un pis-aller, un défaut ou un état de l'écriture par défaut; elle suppose aussi une dynamique d'accompagnement, puisqu'une reprise de cet ordre peut aussi bien avoir lieu au seuil de l'écriture qu'au cours de la préparation du roman. Une telle conception, adaptée à la genèse du roman vernien, permet de se libérer du carcan romantique imposant le génie individuel comme source de l'écriture et les manquements à cette économie scripturale comme des erreurs. Dès lors peuvent apparaître d'autres aspects de cette économie, de reconstruction ou de recyclage, qui entrent en résonance avec l'esthétique globale de Verne. En particulier avec ce que Michel Serres appelle «télémachie 43 » : les Voyages extraordinaires apparaissent en effet à une époque de l'histoire où le globe terrestre, entièrement cartographié et déjà arpenté, ne laisse aux nouveaux explorateurs que le loisir de marcher, à l'instar de Télémaque, sur les traces de leurs Ulysses de pères. Une lecture rejointe par celle de Michel Foucault : «Les romans de Jules Verne, c'est la négentropie du savoir. Non pas la science devenue récréative, mais la re-création à partir du discours uniforme de la science ${ }^{44}$. L L'opposition de la «récréation» et de la «re-création» pointe, en ce qui nous concerne, le défaut de la critique à taxer d'ennuyeuses des descriptions et énumérations «mal intégrées» au roman vernien, qui dans sa fonction première aurait dû sacrifier aux codes narratifs du roman d'aventures à vocation récréative. Le roman vernien pourrait bien être autre chose en effet : une entropie négative, c'est-à-dire une réorganisation - et ses listes une constante récapitulation, un work in progress. La lecture même des listes de Verne serait alors à considérer comme témoignant d'un projet toujours en cours. Cette lecture pourrait valoir aussi bien dans les romans publiés, qui gagneraient à être lus comme non finis, que dans la projection par la lecture d'un état antérieur toujours à reconstruire. Voire dans la projection postérieure, de la lecture à l'écriture, d'un «art de la liste» que certains de ses lecteurs, et Perec en particulier, ont exploité. Le mythe palingénésique de la liste laisserait alors entrevoir sa magie, son imaginaire propre : celui d'une liste unique, courant de toute éternité sous le texte, entre les textes, continuellement reprise, d'un témoin l'autre.

42. Almuth Grésillon, Éléments de critique génétique, Paris, CNRS Éditions, 2016 [1994], p. 282.

43. Michel Serres, Jouvences sur Jules Verne, Paris, Minuit, 1974, p. 11.

44. Michel Foucault, Dits et écrits, Paris, Gallimard, «Quarto», 2001 [1966], t. I, p. 540. 
D'un imaginaire l'autre, il ne s'agit pas de remplacer le premier par le second, ni même de considérer le second comme préférable au premier. Mais de constater que, du vide laissé par les étapes perdues de la confection du roman, la nature de la critique a horreur. Et qu'elle y investit un paradigme d'appréciation, parmi d'autres possibles, soumis au changement ou à l'errance mais susceptible d'être observé et décrit. Et ce, y compris en pensant la projection de cet imaginaire dans une perspective génétique : puisqu'une telle projection se présente d'ores et déjà sous la plume de la critique généraliste, les spécialistes auraient tout intérêt à prendre la question au sérieux, afin d'en rendre compte dans le cadre d'une réflexion génétique, plus informée et plus approfondie que ne le permet un tel article.

Le sérieux documentaire qui préside à la discipline génétique devrait imposer qu'un(e) vrai(e) généticien(ne) ne projette pas d'imaginaire sur les béances de ses dossiers. Mais n'y a-t-il pas, dans cette pratique, quelque chose d'inévitable, et au-delà du cas de Verne, une perspective à interroger, devant toute insuffisance conséquente d'un dossier génétique ? Après tout, $s i$ «la génétique du texte a quelque chose [...] d'une enquête policière 45 », faut-il pour autant déclarer forfait lorsque certains indices manquent? Il me semble que non, pour autant que l'on poursuive la métaphore. Il serait alors nécessaire de considérer comme utile à l'enquête, en parallèle à l'étude factuelle des indices, l'existence d'un faisceau de présomptions. Et de se donner les moyens d'intégrer celui-ci aux outils de la critique : le fait génétique n'est peut-être pas toujours son unique objet. 
GASPARD TURIN est maître assistant à l'université de Lausanne, où il enseigne la littérature moderne et contemporaine, et la critique. Ses recherches portent également sur la didactique de la littérature. Il a publié, en 2017, Poétique et usages de la liste littéraire. Le Clézio, Modiano, Perec, aux éditions Droz. Il est l'auteur de nombreux articles, notamment sur Pascal Quignard, Michel Houellebecq, Éric Chevillard, Marie NDiaye ou encore Georges Perec.

gaspard.turin@unil.ch

\section{Résumés \\ Imaginaire génétique : la critique face à la liste chez Jules Verne}

La critique vernienne tend à considérer les listes, fréquentes dans les Voyages extraordinaires, sous un œil sévère : elles seraient le résultat d'une mauvaise intégration de documents importés d'ouvrages scientifiques, liés au mandat didactique du romancier. Cet enjeu allographe se traduit insidieusement par un jugement exogénétique : les listes présentes dans les romans publiés de Verne seraient dues à un processus d'écriture mal négocié, trop rapide, de l'ordre du recopiage, de la non-écriture. Or une telle hypothèse est invérifiable, puisque les documents qui auraient permis de retracer ces étapes de l'écriture n'ont pas été conservés. Ce que l'on observe alors est le recours de la critique généraliste à un imaginaire génétique, appelé par l'incomplétude du dossier génétique. L'enjeu de cet article, dès lors métacritique, revient à interroger une telle notion et la possibilité de son usage, au sein d'une discipline historiquement consacrée à la matérialité et à la factualité des traces de l'écriture.

Vernian criticism tends to consider lists, which are frequent in the Voyages extraordinaires, unfavorably: they are presumed to be the result of an inept integration of documents imported from scientific books related to the didactic mandate of the novelist. This allographic issue is insidiously translated into an exogenetic judgment: lists in the published novels of Verne, perhaps copied or in any case unwritten, are therefore considered to be due to a poorly negotiated writing process. Such an assumption is unverifiable, since the documents that would have made it possible to trace these stages of writing have not been preserved. What we observe then is the appeal of generalist criticism to a genetic imaginary resulting from the incompleteness of the genetic file. The aim of this article, which is, therefore, metacritical, is to question such a notion as well as the possibility of its use within a discipline historically devoted to the materiality and factuality of traces of writing.

Die Kritik von Verne neigt dazu, die Listen, die in den Voyages extraordinaires häufig vorkommen, unter einem strengen Auge zu betrachten: Sie seien das Ergebnis einer schlechten Integration von aus wissenschaftlichen Büchern importierten Dokumenten, die mit dem didaktischen Auftrag des Romanautors zusammenhängen. Dieses Fremdurteil wird durch eine exogenetische Hypothese unterstützt: Die in den veröffentlichten Romanen von Verne vorhandenen Listen seien auf einen unausgewogenen, zu schnellen Schreibprozess hinsichtlich des Zusammenkopierens, des Nicht-Schreibens zurückzuführen. Eine solche Annahme ist nicht überprüfbar, da die Dokumente, die diese Schreibphasen nachvollziehbar gemacht hätten, nicht erhalten geblieben sind. Was wir dann beobachten, ist der Rückgriff der allgemeinen Kritik auf eine imaginäre Genese die durch die Unvollständigkeit des genetischen Dossiers hervorgerufen wird. Die Herausforderung dieses Artikels, der daher metakritisch ist, besteht darin, eine solche Vorstellung und die Möglichkeit ihrer Verwendung innerhalb einer Disziplin, die sich historisch gesehen der Materialität und Faktizität der Schreibspuren widmet, in Frage zu stellen.
La crítica verniana suele considerar las listas, frecuentes en los Viajes extraordinarios, con una cierta severidad: éstas serían el resultado de una mala integración de documentos importados de tratados científicos, vinculados a la vocación didáctica del novelista. Esta problemática alógrafa se traduce insidiosamente en un juicio exogenético: las listas presentes en las novelas de Verne publicadas provendrían de un proceso de escritura mal efectuado, demasiado rápido, que corresponde a la copia, a la no-escritura. Ahora bien, esta hipótesis resulta inverificable, ya que los documentos que habrían permitido analizar las etapas de esta escritura no se han conservado. Lo que se pone de manifiesto aquí es el uso que hace una crítica no especializada de un imaginario genético, impelido por el carácter incompleto del dossier genético. El objetivo de este artículo -evidentemente metacrítico- es cuestionar esta noción y sus posibilidades de utilización en el seno de una disciplina históricamente dedicada a la materialidad y la factualidad de las huellas de la escritura.

A crítica de Jules Verne tende a encarar com algum rigor as abundantes listas que se encontram em Voyages extraordinaires: elas seriam resultado de uma má integração de documentos importados da literatura científica, relacionada com os propósitos didácticos do romancista. Essa prática alográfica é avaliada, insidiosamente, como exogenética: as listas presentes nos romances publicados de Verne seriam devidas a um processo de escrita mal negociado, demasiado rápido, próximo da cópia e do não-redigido. No entanto, tal hipótese interpretativa não é verificável, por falta dos documentos que teriam permitido rastrear essas etapas da escrita. Trata-se, assim, do recurso da crítica generalista a uma genética imaginária, possibilitada pela incompletude do dossier genético. O fim do presente artigo, que se assume como metacrítico, é questionar semelhante noção e a legitimidade da sua utilização no âmbito de uma disciplina tradicionalmente dedicada à materialidade e à factualidade dos traços escritos.

La critica verniana tende a stigmatizzare le numerose liste presenti nei Voyages extraordinaires: esse sarebbero il risultato di un'integrazione imperfetta di documenti estratti da opere scientifiche, inerenti alla missione didattica del romanziere. Questo problema allografico si traduce insidiosamente in un giudizio esogenetico: le liste presenti nei romanzi di Verne sarebbero dovute a un troppo rapido e impreciso processo di scrittura, assimilabile alla ricopiatura, alla non-scrittura. Una tale ipotesi è tuttavia inverificabile, poiché i documenti che avrebbero permesso di risalire a queste fasi della scrittura non sono stati conservati. Osserviamo dunque il ricorso della critica generalista a un immaginario genetico, convocato dall'incompletezza del dossier genetico. Lo scopo di quest' articolo "meta-critico" è d'interrogare una tale nozione e la possibilità del suo utilizzo in seno a una disciplina storicamente consacrata alla materialità e alla fattualità delle tracce della scrittura. 